\title{
A Robust, Symmetric Operator-Composition Integrator for the Berendsen Temperature-Control Molecular Dynamics Equation
}

\author{
Ikuo Fukuda $^{1 * \dagger}$, Séverine Queyroy ${ }^{2}$, and Haruki Nakamura ${ }^{1}$ \\ ${ }^{1}$ Institute for Protein Research, Osaka University, Osaka 565-0871, Japan \\ 2 Aix Marseille Univ, CNRS, ICR, 13397 MARSEILLE Cedex 20, France
}

The Berendsen equations of motion (EOM) are widely used for controlling the temperature of a target physical system in molecular dynamics simulations. Its numerical integration, however, has never raised much attention. Yet, a non-optimal integration scheme definitely lowers the efficiency of the EOM. If the integration becomes more robust, then does the Berendsen method. To realize this, we propose an operator composition scheme having the following properties: symmetricity, i.e., time reversibility in the original differential equation is kept; systematic, i.e., any higher order of the local accuracy can be attained; robustness, i.e., a new velocity scaling factor is bounded, which enables faster temperature control. Our extended EOM formalism, which provides an invariant function, also helps to observe the numerical error that cannot be detected solely by the temperature controllability. These properties of the proposed method were confirmed by applying it into three molecular systems.

\section{Introduction}

Temperature control in molecular dynamics $(\mathrm{MD})^{1,2)}$ is important to conduct a realistic simulation of a physical system. ${ }^{3,4)}$ There are many algorithms to control the temperature of a given physical system, and they are called thermostats. ${ }^{5-7)}$

Among them, the Nosé-Hoover (NH) thermostat ${ }^{8,9)}$ can generate the BoltzmannGibbs distribution at the target temperature under the ergodic assumption. The structure of the NH equations is basically simple and universal, which allows many extensions (see e.g., Refs. 10-13 for recent work and the references in Refs. 6,14 for earlier work). The NH equations are obtained by adding a force of the form $-\zeta v$ to the Newtonian

\footnotetext{
${ }^{*}$ Correspondence should be addressed: ifukuda@protein.osaka-u.ac.jp

${ }^{\dagger}$ Present address: Graduate School of Simulation Studies, University of Hyogo, Kobe 650-0047, Japan
} 
equations of motion (EOM) that the original physical system, defined by coordinate $x$ and velocity $v$, should obey. Here, the friction coefficient-like quantity is a dynamical variable developing according to $\zeta(t) \propto \int_{0}^{t}\left(K\left(v\left(t^{\prime}\right)\right) / K_{0}-1\right) \mathrm{d} t^{\prime}+$ const., where $K(v)$ and $K_{0}$ are the present and target values of the kinetic energy of the physical system, with $t$ being a time. In this sense the NH equations are based on an "integral" scheme ${ }^{15)}$ for controlling the temperature, or the kinetic energy, where the deviation $K(v) / K_{0}-1$ is integrated with respect to time (we consider the instantaneous temperature and the kinetic energy to be proportional).

The Gaussian isokinetic method ${ }^{16-19)}$ fixes the temperature of the system at the initial value by systematically suppressing the deviations. This is based on the Gauss's constraint method, which can be viewed as a "differential" control scheme, ${ }^{15)}$ since it is defined by the Newtonian EOM attaching the frictional force $-\zeta v$ with $\zeta(t) \propto$ $-\frac{\mathrm{d}}{\mathrm{d} t} U(x(t))$, where $U(x)$ is the potential energy of the physical system.

Berendsen et $a .^{20)}$ proposed an alternative method to control the temperature, based on a "direct" control scheme. This is defined in the same manner as the above methods but uses $\zeta(t) \propto\left(1-K_{0} / K(v(t))\right)$ without integration or differentiation. The Berendsen method is simple, robust, intuitive, and has been employed by many users ${ }^{21)}$ for e.g. biological simulations. The simplicity of the method allows to combine it with a grand canonical $\mathrm{MD}^{22)}$ and with dissipative particle dynamics. ${ }^{23)}$ The robustness, or stability, of the method allows to effectively equilibrate a roughly prepared system or to perform subtle temperature changes in systems for which more elaborated thermostats fail. Although the phase-space distribution produced by the Berendsen method deviates from the canonical distribution, thermodynamic quantities such as the specific heat can be corrected to be those in the canonical or microcanonical MD. ${ }^{24)}$ Furthermore, the Berendsen method, as well as the NH method and the stochastic velocity re-scaling method, ${ }^{25)}$ yield transport properties that are statistically indistinguishable from the ones obtained under the microcanonical ensemble, while the diffusion properties are significantly dampened by the Andersen thermostat and Langevin dynamics, when strong coupling is used. ${ }^{26)}$

In spite of the usefulness of the Berendsen equation, its numerical integration has never raised much attention. The system is not a Hamiltonian system, ${ }^{27,28)}$ so one cannot directly use symplectic integrators, ${ }^{29-33)}$ which has been shown to be efficient in a variety of studies. ${ }^{34-36)}$ This was the main reason to hamper the development of an efficient numerical integration based on a theoretically clear foundation. Most of the 
integration algorithms for the Berendsen EOM are thus based on heuristic approaches, obtained by a combination of the leapfrog method and the velocity scaling, which may give $\mathcal{O}(\Delta t)$ accuracy. However, these approaches lack both the time-reversibility feature and a protocol to attain higher accuracy. Practical reasons may also have prevented the development of an efficient integrator for this EOM. That is, one often supposes that it is sufficient to have a good temperature controllability of the target physical system and that the accuracy is of second importance. However, there are cases where the temperature control is good but a large numerical error is accumulated. Thus a method to capture the error is necessary to get physically correct results.

In this paper we propose a time-reversible (symmetric) integrator of the Berendsen EOM, where the EOM is extended so as to have a time invariant function. These devices are based on the techniques previously developed for non-Hamiltonian systems. ${ }^{37)}$ From the time reversibility, the integrator map preserves the reversible feature that the original ordinary differential equation (ODE) has. This should contribute to the accurate integration. ${ }^{38)}$ By monitoring the value of the constructed invariant function, numerical integration on the extended space can be done without destroying the original solutions of the ODE and will detect the integration errors that cannot be detected by simple temperature monitoring. The integrator is explicit, and furthermore, higher-order integrators can be systematically constructed by the symmetric operator composition technique, which is based on an effective splitting of the target vector field. ${ }^{39)}$ The proposed method also uses a velocity scaling factor that is bounded with respect to $s=h / \tau$, where $\tau$ is the EOM parameter to control the thermostat speed and $h$ is the unit timestep. This new scaling factor has a smaller amplitude than the original one, and the amplitude difference between them increases with $s$. Thus, this new factor limits the unrobustness of the integration and so enhances the stability of the EOM. A larger timestep and smaller $\tau$ can thus be used with the proposed method, allowing a faster temperature control.

We reveal similarities and differences between the proposed and conventional methods both theoretically and numerically. We believe the current study to be the first one to discuss both the Berendsen's method and the integrator mathematics. Section 2 reviews the Berendsen EOM and its integration schemes found in the literature. In Sect. 3, we present our integration scheme and demonstrate its fundamental properties. In Sect. 4 we theoretically discuss the relationship between the proposed and the conventional integration methods. Their mathematical details are demonstrated in Appendix 
and Supplementary material. ${ }^{40)}$ In Sect. 5, we investigate properties of the proposed method and compare it with other methods, via numerical simulations using one model system and two bulk molecular systems. Section 6 summarizes the current work and gives remarks.

\section{Berendsen equations of motion}

The Berendsen EOM can be represented by ${ }^{20)}$

$$
\left.\begin{array}{l}
\dot{x}=v, \\
\dot{v}=F(x) \mathbf{M}^{-1}+\frac{1}{2 \tau}\left(\frac{K_{0}}{K(v)}-1\right) v,
\end{array}\right\}
$$

where $x \equiv\left(x_{1}, \ldots, x_{n}\right) \in D \subset \mathbb{R}^{n}, v \equiv\left(v_{1}, \ldots, v_{n}\right) \in \mathbb{R}^{n}, F(x) \in \mathbb{R}^{n}$, and $K(v) \equiv$ $\sum_{i=1}^{n} m_{i} v_{i}^{2} / 2$ represent the atomic coordinates, velocities, force (smooth vector-valued function on a domain $D$ ), and kinetic energy, respectively, of a physical system of $n$ degrees of freedoms, with $m_{i}$ being a mass parameter, which defines the matrix $\mathbf{M} \equiv \operatorname{diag}\left(m_{1}, \ldots, m_{n}\right)$. The friction-coefficient variable $\zeta \equiv-\frac{1}{2 \tau}\left(\frac{K_{0}}{K(v)}-1\right)$ governs the control of the temperature of a physical system, $T(v) \equiv 2 K(v) / n k_{\mathrm{B}}$, referring to the target temperature $T_{0} \equiv 2 K_{0} / n k_{\mathrm{B}}>0$, (viz., $K_{0}$ is the target kinetic energy value), with $k_{\mathrm{B}}$ being Boltzmann's constant. The parameter (time constant) $\tau>0$ adjusts the control strength, where a large $\tau$ gives a weak control, and the limit $\tau \rightarrow \infty$ reduces to the Newtonian equations of motion. Note that a similar but alternative definition of $\zeta$ is discussed in Ref. 41.

Typical numerical integration schemes to solve the EOM are the following. In the original approach, ${ }^{20,21)}$ the current-timestep coordinates and velocities $\left(x^{\prime}, v^{\prime}\right)$ are obtained from the previous-timestep quantities $(x, v)$ as:

$$
\begin{aligned}
\tilde{v} & =v+h F(x) \mathbf{M}^{-1}, \\
v^{\prime} & =\lambda_{h}(v) \tilde{v}, \\
x^{\prime} & =x+h v^{\prime},
\end{aligned}
$$

where $h$ indicates the unit timestep used in the integration. Here,

$$
\lambda_{h}(v) \equiv\left[1+\frac{h}{\tau}\left(\frac{K_{0}}{K(v)}-1\right)\right]^{\frac{1}{2}}
$$

is the scaling factor of the atomic velocity for the temperature control. ${ }^{20)}$ Note that within scheme (2), it is possible to use another definition of the current-timestep velocity, such as $\left(v^{\prime}+v\right) / 2$, but we will not use it hereafter. Equation (2) is considered to be a 
first-order algorithm (see Sect. 4.2), and we denote it by Method 1 for future reference. Alternatively, we can use a slightly modified version where $\lambda_{h}(\tilde{v})$ is used instead of $\lambda_{h}(v)$ in $(2 \mathrm{~b})$, and we call this the "modified Berendsen scheme" or, simply, Method 1 mod. Note that the original paper $^{20)}$ uses $T_{0} / T(v)$, the ratio of the target temperature and the present temperature, instead of $K_{0} / K(v)$, and defines the linear transformation between these quantities such as $T(v)=2 K(v) / k_{\mathrm{B}}\left(3 N-N_{\mathrm{c}}-3\right)$, with $N$ being the number of atoms and $N_{\mathrm{c}}$ being the number of constraints. We see that $K_{0} / K(v)=T_{0} / T(v)$ irrespective of the linear transformation, so that (1) and (3) are suitable.

As considered in Khalili et al., ${ }^{42)}$ the velocity scaling and the Verlet scheme can be combined such as

$$
\begin{aligned}
x^{\prime} & =x+h v+\frac{h^{2}}{2} F(x) \mathbf{M}^{-1}, \\
\tilde{v} & =v+\frac{h}{2}\left(F(x)+F\left(x^{\prime}\right)\right) \mathbf{M}^{-1}, \\
v^{\prime} & =\lambda_{h}(\tilde{v}) \tilde{v} .
\end{aligned}
$$

Namely, the scaling is done after the velocity Verlet algorithm; we call it Method 2. An alternative choice is to use $\lambda_{h}(v)$ instead of $\lambda_{h}(\tilde{v})$ in $(4 \mathrm{c})$.

\section{Extended system and integration scheme}

\subsection{Extended ODE and invariant}

The simple scheme ${ }^{37)}$ to construct an invariant function is briefly reviewed in Sect. 3.1.1, and it is applied to the Berendsen EOM in Sect. 3.1.2.

\subsubsection{General scheme}

For a given arbitrary smooth ODE in a domain $\Omega$ of $\mathbb{R}^{N}$,

$$
\dot{\omega}=X(\omega)
$$

we associate an additional variable $\mathrm{v} \in \mathbb{R}$ to the original variables $\omega=\left(\omega_{1}, \ldots, \omega_{N}\right) \in \Omega$ and represent them by $\omega^{\prime}=(\omega, \mathrm{v})$ as a point of an "extended space" $\Omega^{\prime} \equiv \Omega \times \mathbb{R}$. We then make an "extended ODE" ${ }^{37)}$ on $\Omega^{\prime}$,

$$
\dot{\omega}^{\prime}=X^{\prime}\left(\omega^{\prime}\right)
$$

which is defined by

$$
\begin{aligned}
& \dot{\omega}=X(\omega), \\
& \dot{\mathrm{v}}=Y(\omega) .
\end{aligned}
$$


Here $Y: \Omega \rightarrow \mathbb{R}$ is an extended-field function defined by

$$
\begin{aligned}
Y(\omega) & \equiv-(X(\omega) \mid \nabla B(\omega)) \\
& =-\sum_{i=1}^{N} X_{i}(\omega) D_{i} B(\omega),
\end{aligned}
$$

with $B$ being an arbitrary smooth function on $\Omega$. It is then shown that a function

$$
L: \Omega^{\prime} \rightarrow \mathbb{R}, \omega^{\prime} \stackrel{\mathrm{d}}{\longmapsto} B(\omega)+\mathrm{v}
$$

becomes an invariant of the extended ODE; i.e., for an arbitrary solution $\phi^{\prime} \equiv(\omega, \mathrm{v})$ of $(6)$

$$
L\left(\phi^{\prime}(t)\right)=B(\omega(t))+\mathrm{v}(t)
$$

is constant for any time $t$. Thus, by monitoring the conservation of the invariant while numerically integrating the extended ODE, we can check the numerical error. It is clear that all solutions, $t \mapsto \omega(t)$, in the original ODE (7a) are unaffected by adding $\mathrm{v}$ and its $\operatorname{EOM}(7 \mathrm{~b})$.

\subsubsection{For the Berendsen EOM}

According to the scheme, for the Berendsen ODE (1)

$$
\dot{\omega}=X_{\mathrm{B}}(\omega)
$$

where

$$
\begin{aligned}
X_{\mathrm{B}}: \Omega & \rightarrow \mathbb{R}^{2 n}, \\
\omega & \equiv(x, v) \mapsto\left(v, F(x) \mathbf{M}^{-1}+\frac{1}{2 \tau}\left(\frac{K_{0}}{K(v)}-1\right) v\right)
\end{aligned}
$$

with $\Omega \equiv D \times \mathbb{R}_{\times}^{n}\left[\right.$ viz., all $(x, v)$ except $\left.v=0 \in \mathbb{R}^{n}\right]$, the extended ODE is defined by

$$
\begin{aligned}
\dot{\omega}^{\prime} & =X_{\mathrm{B}}^{\prime}\left(\omega^{\prime}\right) \in \mathbb{R}^{2 n+1} \\
& =\left(X_{\mathrm{B}}(\omega), Y(\omega)\right) \\
& =\left(v, F(x) \mathbf{M}^{-1}+\frac{1}{2 \tau}\left(\frac{K_{0}}{K(v)}-1\right) v, Y(\omega)\right),
\end{aligned}
$$

viz.,

$$
\left.\begin{array}{rl}
\dot{x} & =v \\
\dot{v} & =F(x) \mathbf{M}^{-1}+\frac{1}{2 \tau}\left(\frac{K_{0}}{K(v)}-1\right) v, \\
\dot{\mathrm{v}} & =Y(\omega)=-(X(\omega) \mid \nabla B(\omega)),
\end{array}\right\}
$$


and the invariant is $L(\omega, \mathrm{v})=B(\omega)+\mathrm{v}$.

Among a variety of choices of the function $B$, the following one may be physically natural:

$$
B(x, v) \equiv U(x)+K(v)
$$

viz., $B$ is the total energy of the system, where we assume the existence of the potential function $U$ such that $F=-\nabla U$. Applying (14) to (8), we get $Y(\omega)=\frac{1}{\tau}\left(K(v)-K_{0}\right)$ and so have the extended equation and the invariant as follows:

$$
\begin{aligned}
\dot{\mathrm{v}} & =\frac{1}{\tau}\left(K(v)-K_{0}\right), \\
L(\omega, \mathrm{v}) & =U(x)+K(v)+\mathrm{v} .
\end{aligned}
$$

We can also confirm (16) to be a time invariant for (13) by a straightforward differentiation with respect to time:

$$
-(F(x(t)) \mid v(t))+\frac{d}{d t} K(v(t))+\frac{1}{\tau}\left(K(v(t))-K_{0}\right)=0 .
$$

Interestingly, this is equivalent to the relation based on the original consideration of the "global coupling" [i.e., Eq. (9) in Ref. 20]. Note also that as $\tau \rightarrow \infty$, we have the Newtonian limit: the EOM approaches the Newtonian EOM, and the invariant (16) approaches the Newtonian total energy $K(v)+U(x)$ up to $\mathrm{v}(0)=$ const.

A slightly generalized choice of $B$ defined by

$$
B(x, v) \equiv c_{1} U(x)+c_{2} K(v),
$$

where $c_{1}$ and $c_{2}$ are parameters, produces the following EOM of $\mathrm{v}$ and the invariant,

$$
\begin{aligned}
\dot{\mathrm{v}} & =Y(\omega) \\
& =\left(c_{1}-c_{2}\right)(F(x) \mid v)+\frac{c_{2}}{\tau}\left(K(v)-K_{0}\right), \\
L(\omega, \mathrm{v}) & =c_{1} U(x)+c_{2} K(v)+\mathrm{v},
\end{aligned}
$$

respectively. It is pointed out that the choice of $c_{1}=0$ would be useful in the case where the potential $U$ does not exist.

Note that Bussi et al. ${ }^{25)}$ proposed a stochastic canonical sampling method along the line of the velocity scaling and discussed an associated conserved quantity that is defined for an individual trajectory. The notion of this conserved quantity seems similar to that of the extended invariant ${ }^{37)}$ for an ODE. However, our target here is in the ODE, and the invariant is a function globally defined in the phase space, in contrast to the 
approach of Bussi et al.

\subsection{Integrator}

\subsubsection{First-order Integrator}

To construct a numerical integrator, we decompose a target vector field and compose the corresponding phase space maps, according to the scheme described in Ref. 37. We decompose the target extended field $X_{\mathrm{B}}^{\prime}$, defined by $(12 \mathrm{~b})-(12 \mathrm{c})$, as $X_{\mathrm{B}}^{\prime}=\sum_{i=1}^{4} X^{\prime[i]}$, where

$$
\begin{aligned}
X^{\prime[1]}\left(\omega^{\prime}\right) & \equiv(v, 0,0), \\
X^{\prime[2]}\left(\omega^{\prime}\right) & \equiv\left(0, F(x) \mathbf{M}^{-1}, 0,\right), \\
X^{\prime[3]}\left(\omega^{\prime}\right) & \equiv\left(0, \frac{1}{2 \tau}\left(\frac{K_{0}}{K(v)}-1\right) v, 0\right), \\
X^{\prime[4]}\left(\omega^{\prime}\right) & \equiv(0,0, Y(\omega)) .
\end{aligned}
$$

The point to get the decomposition is to ensure that each ODE

$$
\dot{\omega}^{\prime}=X^{\prime[i]}\left(\omega^{\prime}\right)
$$

can be solved explicitly. This is trivial for $i=1,2$, and 4 , but may not be for $i=3$ :

$$
\left.\begin{array}{rl}
\dot{x} & =0, \\
\dot{v} & =\frac{1}{2 \tau}\left(\frac{K_{0}}{K(v)}-1\right) v \\
\dot{\mathrm{v}} & =0 .
\end{array}\right\}
$$

We find that the solution of ODE (22) taking an initial value $\left(x_{0}, v_{0}, \mathrm{v}_{0}\right) \in \Omega^{\prime}$ is

$$
\begin{aligned}
& x(t)=x_{0}, \\
& v(t)=\left[\left(1-\frac{K_{0}}{K\left(v_{0}\right)}\right) \exp \left(-\frac{t}{\tau}\right)+\frac{K_{0}}{K\left(v_{0}\right)}\right]^{\frac{1}{2}} v_{0}, \\
& \mathrm{v}(t)=\mathrm{v}_{0} .
\end{aligned}
$$

It should be stressed that $X^{\prime[3]}$ is a smooth (now, of class $C^{\infty}$ ) field on $\Omega^{\prime}$ so that the solution of the initial value problem is unique. Since we can directly check that (23) satisfies both $(22)$ and $(x(0), v(0), \mathrm{v}(0))=\left(x_{0}, v_{0}, \mathrm{v}_{0}\right)$, Eq. (23) is the unique solution. Note that the decomposition of $X_{\mathrm{B}}^{\prime}$ is natural in that the additional quantities $\mathrm{v}$ and $Y$ do not affect the solutions of a decomposed original ODE $\dot{\omega}=X^{[i]}(\omega)$ for $i=1,2$, and 3 , where $X^{[i]}$ is obtained by the same type of the decomposition of the original (not extended) field $X_{\mathrm{B}}=\sum_{j=1}^{3} X^{[i]}$ [viz., $X^{[i]}$ is defined by removing 0 in the last column 
of (20a), (20b), and (20c) for $i=1,2$, and 3, respectively]. In particular, (23) is not affected by $\mathrm{v}$ and $Y$.

Hence, the exact flow $\Phi_{t}^{[i]}$ for each vector field $X^{\prime[i]}$, where $t \mapsto \Phi_{t}^{[i]}\left(\omega^{\prime}\right)$ denotes the solution of (21) with an initial value $\omega^{\prime} \in \Omega^{\prime}$, is thus represented by the following map or operator on the extended phase space $\Omega^{\prime}$ :

$$
\begin{aligned}
& \Phi_{h}^{[1]}: \omega^{\prime} \mapsto(h v+x, v, \mathrm{v}), \\
& \Phi_{h}^{[2]}: \omega^{\prime} \mapsto\left(x, h F(x) \mathbf{M}^{-1}+v, \mathrm{v}\right), \\
& \Phi_{h}^{[3]}: \omega^{\prime} \mapsto\left(x, \Lambda_{h}(v) v, \mathrm{v}\right), \\
& \Phi_{h}^{[4]}: \omega^{\prime} \mapsto(x, v, h Y(x, v)+\mathrm{v}),
\end{aligned}
$$

where we have used $h$ instead of $t$. Here,

$$
\Lambda_{h}(v) \equiv\left[\left(1-\frac{K_{0}}{K(v)}\right) \exp \left(-\frac{h}{\tau}\right)+\frac{K_{0}}{K(v)}\right]^{\frac{1}{2}}
$$

comes from (23b) and becomes the counterpart of $\lambda_{h}(v)$ defined in (3); see the next section for their comparison and see Appendix for their detailed properties.

Then we get a first-order integrator with an unit timestep $h$,

$$
\Phi_{h}=\Phi_{h}^{[4]} \circ \Phi_{h}^{[3]} \circ \Phi_{h}^{[2]} \circ \Phi_{h}^{[1]},
$$

which will be a map from $\Omega^{\prime}$ to $\Omega^{\prime}$ (see Sect. IV of Supplementary material ${ }^{40}$ ) for mathematical details, including the fact that the maps $\Phi_{h}^{[i]}$ are not necessarily defined for all $h \in \mathbb{R}$ except for $i=4)$. There are many possibilities ${ }^{37)}$ about the appearing order of $\Phi_{h}^{[i]}$ in (26), and we discuss it later. The integration scheme (26), defining the change from the preceding values to the present values, $(x, v, \mathrm{v}) \mapsto\left(x^{\prime}, v^{\prime}, \mathrm{v}^{\prime}\right)$, is also expressed in an explicit operation form,

$$
\begin{aligned}
x^{\prime} & =x+h v, \\
\tilde{v} & =h F\left(x^{\prime}\right) \mathbf{M}^{-1}+v, \\
v^{\prime} & =\Lambda_{h}(\tilde{v}) \tilde{v}, \\
\mathrm{v}^{\prime} & =h Y\left(x^{\prime}, v^{\prime}\right)+\mathrm{v},
\end{aligned}
$$

and practically used in computer code. Rather than the form of these operations, the form of (26) expressed by the maps $\Phi_{h}^{[i]}$ is helpful for theoretical analyses. The latter form, which has not been taken into account in conventional studies for the Berendsen EOM though, enables us to properly grasp $\Phi_{h}^{[i]}, \Phi_{h}$, and $T_{h}$, where $\Phi_{h}^{[i]}(i=1, \ldots, 4)$ 
are the constituents of the integration map $\Phi_{h}$, which in turn mimic the exact flow $T_{h}$ for the target $\operatorname{ODE}(12) ; \Phi_{h}^{[i]}, \Phi_{h}$, and $T_{h}$ are uniformly viewed as maps (operators) on $\Omega^{\prime}$ and can be analyzed with certain properties of maps (the so-called "symplectic integrator" on a Hamiltonian system ${ }^{28)}$ is constructed in the same spirit, where the symplectic property of maps is concerned).

\subsubsection{Higher-order Integrator}

The viewpoint of the maps is also useful to systematically raise the (local) accuracy of the integrator. In fact, an integrator with second-order accuracy can be constructed as a map by composing maps based on $\Phi_{h}$, which is of the first-order accuracy. Here, an integrator map $\Psi_{h}$ is said to be $p$ th-order, if

$$
T_{h}\left(\omega^{\prime}\right)-\Psi_{h}\left(\omega^{\prime}\right)=\mathcal{O}\left(h^{p+1}\right)
$$

holds for any $\omega^{\prime}$. A typical second-order integrator is an extended version of the Verlet method: ${ }^{37,43)}$

$$
\Psi_{h}=\Phi_{h / 2} \circ \Phi_{h / 2}^{*},
$$

where

$$
\begin{aligned}
\Phi_{t}^{*} & \equiv\left(\Phi_{-t}\right)^{-1} \\
& =\Phi_{h}^{[1]} \circ \Phi_{h}^{[2]} \circ \Phi_{h}^{[3]} \circ \Phi_{h}^{[4]}
\end{aligned}
$$

is the adjoint map of $\Phi_{t}$ and also a first-order integrator. Thus we have

$$
\Psi_{h}=\Phi_{h / 2}^{[4]} \circ \Phi_{h / 2}^{[3]} \circ \Phi_{h / 2}^{[2]} \circ \Phi_{h}^{[1]} \circ \Phi_{h / 2}^{[2]} \circ \Phi_{h / 2}^{[3]} \circ \Phi_{h / 2}^{[4]} .
$$

Equations (30b) and (31) can be automatically derived from the fact that each map $\Phi_{h}^{[i]}$ is the exact flow [of the decomposed ODE (21)]. The explicit forms of operations for $\Psi_{h}$ are provided by the similar manner as (27).

The appearing order of $\Phi_{h}^{[j]}(j=1, \ldots, 4)$ in $(26)$ is arbitrary to ensure the firstorder local accuracy. However, this ordering has an influence on the computational time needed. The most time-consuming operand in (24) is the evaluation of force $F(x)$ or potential $U(x)$. The force evaluation is required for $\Phi_{h}^{[2]}$. In addition, the evaluation of $F(x)$ or $U(x)$ may be required for $\Phi_{h}^{[4]}$ (function $Y$ ) and for the invariant. Here, specifically consider the typical integration form (29). Then, the number of the force or potential evaluation is 1 (viz., the minimum) for any cases if we use the ordering of (26), but otherwise it may be 2. Details are discussed in Sect. I of Supplementary 
material. ${ }^{40)}$ For these reasons, one of our recommended ordering, for any $Y$, is given by (26).

Now, using the first-order maps defined by (26) and (30), higher-order integrators can be obtained as a map by the symmetric composition with the adjoint: ${ }^{44)}$

$$
\Psi_{h}=\Phi_{\alpha_{s} h} \circ \Phi_{\beta_{s} h}^{*} \circ \cdots \circ \Phi_{\alpha_{2} h} \circ \Phi_{\beta_{2} h}^{*} \circ \Phi_{\alpha_{1} h} \circ \Phi_{\beta_{1} h}^{*},
$$

where coefficients $\left\{\alpha_{i}, \beta_{i}\right\} \subset \mathbb{R}$ satisfy the symmetric condition

$$
\alpha_{i}=\beta_{s+1-i}, \quad i=1, \ldots, s,
$$

in order to satisfy the symmetric property: $\Psi_{h}^{*}=\Psi_{h}$ [see also Sect. 4.3 (ii)]. Specific values of the parameters, viz., stage $s$ and coefficients $\left\{\alpha_{i}, \beta_{i}\right\}$, as described in Ref. 43 , can be used, and we have presented several second-order integrators (they are designated as P2S1 or P2S2 ${ }^{44,45)}$ ) and fourth-order integrators $\left(\mathrm{P} 4 \mathrm{~S} 5^{45)}\right.$ and $\left.\mathrm{P} 4 \mathrm{~S} 6^{46)}\right)$. See also Sect. 7.3 for more details on technical aspects. Among second-order integrators, the simplest one is called as P2S1, defined by (29):

$$
\Psi_{h}^{\mathrm{P} 2 \mathrm{~S} 1}=\Phi_{h / 2} \circ \Phi_{h / 2}^{*} .
$$

Note that a volume preserving integrator can be constructed, according to the scheme proposed here, using the twisting technique described in Ref. 37.

\section{Relationships between the integrators}

\subsection{Reinterpretation of the conventional methods}

To observe the relationship between the proposed and the conventional integration methods, we first consider the velocity scale factors $\Lambda_{h}(v)$ and $\lambda_{h}(v)$, which are given by (25) and (3), respectively. They are intimately related with each other. As can be obtained by expansions of functions, we get

$$
\begin{aligned}
\Lambda_{h}(v) & =\lambda_{h}(v)+\mathcal{O}\left(h^{2}\right) \\
& =\lambda_{h}(v)+\mathcal{O}\left((h / \tau)^{2}\right) .
\end{aligned}
$$

Equation (34b) indicates the similarity of $\Lambda_{h}(v)$ and $\lambda_{h}(v)$ when $h \ll \tau$, viz., when the timestep is several orders of magnitude smaller than the temperature-control parameter (see Appendix for more detailed comparisons). Thus, the factor $\lambda_{h}(v)$, which was originally introduced in the Method $1,{ }^{20}$ can also be derived as an approximation of the factor $\Lambda_{h}(v)$ that appears as a component of $\Phi_{h}^{[3]}$ [Eq. (24c)], which is the exact flow of the decomposed Berendsen field, $X^{\prime[3]}[\mathrm{Eq}$. (20c)], which can also be identified with 
$X^{[3]}$, as stated. In other words, $\Lambda_{h}(v)$ is very natural for the original Berendsen EOM.

Equation (34) implies that the conventional methods can be seen as certain approximations, with respect to $h$, of the proposed method. To clarify this, first notice, as implied from (34a) (see Sect. IV of Supplementary material ${ }^{40)}$ ), the fact that

$$
\Phi_{h}^{[3]}\left(\omega^{\prime}\right)=\tilde{\Phi}_{h}^{[3]}\left(\omega^{\prime}\right)+\mathcal{O}\left(h^{2}\right)
$$

where

$$
\tilde{\Phi}_{h}^{[3]}\left(\omega^{\prime}\right) \equiv\left(x, \lambda_{h}(v) v, \mathrm{v}\right) .
$$

Using these terminologies and related ones, we can redefine the conventional integrators as maps on the extended space. First, Method 2 [Eq. (4)] can be represented as

$$
\Phi_{\mathrm{M} 2, h} \equiv \Phi_{h}^{[4]} \circ \tilde{\Phi}_{h}^{[3]} \circ \Phi_{h / 2}^{[2]} \circ \Phi_{h}^{[1]} \circ \Phi_{h / 2}^{[2]} .
$$

If we ignore the extended variable $\mathrm{v}$, then (37) is completely consistent with (4). Here, $\mathrm{v}$ does not affect, as well as the original EOM, the development of $x$ and $v$ defined by the original Method 2, and v is changed (boosted) only by $\Phi_{h}^{[4]}$. Thus, $\Phi_{h}^{[4]}$ can be freely composed and we may define e.g., $\Phi_{\mathrm{M} 2, h} \equiv \tilde{\Phi}_{h}^{[3]} \circ \Phi_{h / 2}^{[2]} \circ \Phi_{h}^{[1]} \circ \Phi_{h / 2}^{[2]} \circ \Phi_{h}^{[4]}$ instead. The reason why we choose (37) is that we can take a similar form as our basic first-order map (26) [viz., $\Phi_{h}^{[4]}$ is placed at the last] to conduct their comparisons. This choice will also apply to other maps described below.

Similarly, Method 1 mod can be represented as

$$
\Phi_{\mathrm{M} 1 \mathrm{~m}, h} \equiv \Phi_{h}^{[4]} \circ \Phi_{h}^{[1]} \circ \tilde{\Phi}_{h}^{[3]} \circ \Phi_{h}^{[2]} .
$$

Recall that Method $1 \bmod$ is a modification of Method 1 [Eq. (2)], which can now be represented by

$$
\Phi_{\mathrm{M} 1, h} \equiv \Phi_{h}^{[4]} \circ \Phi_{h}^{[1]} \circ \check{\Phi}_{h}^{[3]} \circ \Phi_{h}^{[2]},
$$

where

$$
\check{\Phi}_{h}^{[3]}\left(\omega^{\prime}\right) \equiv\left(x, \lambda_{h}\left(v-h F(x) \mathbf{M}^{-1}\right) v, \mathrm{v}\right) .
$$

$\check{\Phi}_{h}^{[3]}$ is similar to $\tilde{\Phi}_{h}^{[3]}$ but not equivalent, and reflects the fact that in Method 1 the velocity before the mapping by $\Phi_{h}^{[2]}$ is referred as an argument of $\lambda_{h}$. Note that we have included $\Phi_{t}^{[4]}$ in $\Phi_{\mathrm{M} 1 \mathrm{~m}, h}$ and $\Phi_{\mathrm{M} 1, h}$ using the same concept as that in $\Phi_{\mathrm{M} 2, h}$. 


\subsection{Similarity}

Now, we can show, as detailed in Sect. IV of Supplementary material, ${ }^{40)}$ that these three maps, $\Phi_{\mathrm{M} 1 \mathrm{~m}, h}, \Phi_{\mathrm{M} 1, h}$, and $\Phi_{\mathrm{M} 2, h}$, are equivalent to $\Phi_{h}$ in the first-order accuracy, where $\Phi_{h}$ is a currently given map defined by (26). Namely,

$$
\begin{aligned}
\Phi_{\mathrm{M} 1 \mathrm{~m}, h}\left(\omega^{\prime}\right) & =\Phi_{h}\left(\omega^{\prime}\right)+\mathcal{O}\left(h^{2}\right) \\
& =\left(\Phi_{h}^{[4]} \circ \Phi_{h}^{[3]} \circ \Phi_{h}^{[2]} \circ \Phi_{h}^{[1]}\right)\left(\omega^{\prime}\right)+\mathcal{O}\left(h^{2}\right), \\
\Phi_{\mathrm{M} 1, h}\left(\omega^{\prime}\right) & =\Phi_{h}\left(\omega^{\prime}\right)+\mathcal{O}\left(h^{2}\right), \\
\Phi_{\mathrm{M} 2, h}\left(\omega^{\prime}\right) & =\Phi_{h}\left(\omega^{\prime}\right)+\mathcal{O}\left(h^{2}\right) .
\end{aligned}
$$

As an intermediate type between $\Phi_{\mathrm{M} 1 \mathrm{~m}, h}$ and $\Phi_{h}$, a map,

$$
\tilde{\Phi}_{h} \equiv \Phi_{h}^{[4]} \circ \tilde{\Phi}_{h}^{[3]} \circ \Phi_{h}^{[2]} \circ \Phi_{h}^{[1]},
$$

which uses $\tilde{\Phi}_{h}^{[3]}$ instead of $\Phi_{h}^{[3]}$ in $\Phi_{h}$, can be defined and is also equivalent to $\Phi_{h}$ in the first order:

$$
\tilde{\Phi}_{h}\left(\omega^{\prime}\right)=\Phi_{h}\left(\omega^{\prime}\right)+\mathcal{O}\left(h^{2}\right) .
$$

Since $\Phi_{h}$ is a first-order map, the above four maps become first-order integrators. Note that, however, this does not straightforwardly indicate that the maps do not have higher-order property. In particular, $\Phi_{\mathrm{M} 2, h}$ includes the part, $\Phi_{h / 2}^{[2]} \circ \Phi_{h}^{[1]} \circ \Phi_{h / 2}^{[2]}$, which corresponds to the second-order map for the Newtonian EOM, so that $\Phi_{\mathrm{M} 2, h}$ may behave as a second-order map if the Newtonian parts are dominant, as will be discussed later.

Computational cost of the current first-order map $\Phi_{h}$ and that of the conventional maps are essentially the same. In addition, the difference in the cost between the current second-order P2S1 map [see (29) or (31)] and the conventional maps is at most $\mathcal{O}(n)$, which can be mostly ignored against the $\mathcal{O}\left(n^{2}\right)$-cost generally required in the force evaluation for $\Phi_{h}^{[2]}$. This is the consequence of the choice of the appearing order of $\Phi_{h}^{[j]}$, as discussed. However, note also that the cost becomes higher if we use higher-order integrators, in general.

\subsection{Difference}

As stated, the conventional integrators, including the Berendsen's map $\Phi_{\mathrm{M} 1, h}$ and the related map $\Phi_{\mathrm{M} 1 \mathrm{~m}, h}$, are equivalent to the current 1st-order scheme $\Phi_{h}$ in the lowestorder local accuracy. However, the current whole algorithm (32), using $\Phi_{h}$ and $\Phi_{h}^{*}$, is not the same as the conventional methods, so that the overall behavior should not also 
be the same. The following three differences are given:

\section{(i) Order of the local accuracy-}

Equation (32) is at least second order (which is the same as that of the Verlet method), while the conventional ones, $\Phi_{\mathrm{M} 1, h}$ and $\Phi_{\mathrm{M} 1 \mathrm{~m}, h}$, are 1st order. This difference holds even when the current algorithm $(32)$ uses $\tilde{\Phi}_{h}^{[3]}$, instead of $\Phi_{h}^{[3]}$.

\section{(ii) Time reversibility (symmetric property)-}

Here, a map $\phi_{h}$ parametrized by a time parameter $h$ is said to be time reversible (at $h$ ), if $\phi_{-h} \circ \phi_{h}$ is well defined and becomes the identity; namely, if we go forward and then back with $h$, then we get to the starting point. Note that this is a fundamental property of all solutions of arbitrary smooth autonomous ODEs, including the original Berendsen EOM (1). Time reversibility is often referred as a self-adjoint property ${ }^{38)}$ or symmetric property (here, we define the latter as a slightly stronger property than the former): $\phi_{h}$ is said to be symmetric (at $h$ ), if $\phi_{h}^{*}$ exists and $\phi_{h}^{*}=\phi_{h}$. Since a numerical integrator mimics the exact solutions of the original ODE, it should be time reversible. [Here, the time reversibility does not mean the $\rho$-reversibility, ${ }^{44)}$ which is considered for a vector field $X$ satisfying $\rho \circ X \circ \rho^{-1}=-X$ for an invertible linear map $\rho$ such as $(x, v) \mapsto(x,-v)$.] We see that $\Psi_{h}[$ Eq. (32)] is symmetric provided that the parameters obey (33). In contrast, the conventional ones, $\Phi_{\mathrm{M} 1, h}, \Phi_{\mathrm{M} 2, h}$, and $\Phi_{\mathrm{M} 1 \mathrm{~m}, h}$, are not. Note also that $\Phi_{h}^{[i]}(i=1, \ldots, 4)$ used in the current integrator are symmetric (time reversible), but $\tilde{\Phi}_{h}^{[3]}$ used in conventional integrators is not in general (where the usual group property is lost).

\section{(iii) Robustness-}

The scaling factors $\lambda_{h}(v)$ [Eq. (3)] and $\Lambda_{h}(v)$ [Eq. (25)] fluctuate according to the change of the kinetic energy $K(v)$ during the simulation; they are decreasing with increasing $K(v)$ for $h>0$. The method using $\Lambda_{h}(v)$ is more robust than the method using $\lambda_{h}(v)$ (mathematical details are shown in Appendix) for two reasons. (1) First, the amplitude of $\lambda_{h}(v)$ is larger than that of $\Lambda_{h}(v)$ for any $K(v)$ and any $h>0$. This indicates that we often have to treat significant changes for $\lambda_{h}(v)$ in general. Since this comparison is for the same $K(v)$ value but not for the same simulation step, the above indication makes sense as long as the dynamics obtained by using $\Lambda_{h}(v)$ encounters similar or smaller fluctuations of $K(v)$ than those obtained by using $\lambda_{h}(v)$. (2) Second, when the factor $h / \tau$ becomes large, $\Lambda_{h}(v)$ remains finite, because the factor appears 
only in the exponential term, while $\lambda_{h}(v)$ can become very large, leading to instabilities. Namely, the difference of the two amplitudes, $\left|\lambda_{h}(v)-\Lambda_{h}(v)\right|$, grows with increasing $h / \tau$. The scheme using $\Lambda_{h}(v)$ is, thus, more robust than the one using $\lambda_{h}(v)$, as we increase $h$ to use a larger timestep (to reduce overall computer time) and/or decrease $\tau$ (to control the temperature faster). Here, note that the parameters $\tau$ and $h / \tau$ appear only in $\Phi_{h}^{[3]}, \tilde{\Phi}_{h}^{[3]}$, and $\check{\Phi}_{h}^{[3]}$, through $\lambda_{h}(v)$ and $\Lambda_{h}(v)$.

\section{Numerical Simulations}

Among the proposed integrators, we examined the most fundamental one, P2S1, in detail and compared it with the conventional methods, Method 1, Method 1 mod, and Method 2. A basic model system and two bulk systems were used for the examinations. All the simulations were performed with a program specially developed for this study.

\subsection{Material}

(1) Isolated ethane molecule. The molecule is designed by two $\mathrm{CH}_{3}$ united atoms $\left(m_{1}=m_{2}=15 \mathrm{~g} / \mathrm{mol}\right)$ and one harmonic spring connecting the two united atoms. The interaction is thus $U(r)=k\left(r-r_{0}\right)^{2}$, where $r$ is the distance between the atoms, $r_{0}$ is its equilibrium value, and $k$ is the spring constant. The parameters used were $r_{0}=1.54 \AA$ and $k=240 \AA^{-2} \mathrm{kcal} / \mathrm{mol}$, and the target temperature $T_{0}$ was $300 \mathrm{~K}$, as set in Ref. 47 . The initial coordinates $x(0) \equiv\left(\boldsymbol{x}_{1}(0), \boldsymbol{x}_{2}(0)\right)$ and velocities $v(0) \equiv\left(\boldsymbol{v}_{1}(0), \boldsymbol{v}_{2}(0)\right)$ were $\boldsymbol{x}_{1}(0)=-\boldsymbol{x}_{2}(0)=(-0.8,0,0)$ and $\boldsymbol{v}_{1}(0)=-\boldsymbol{v}_{2}(0)=(\sigma, 0,0)$, where $\sigma=\sqrt{3 k_{\mathrm{B}} T_{0} / m_{1}}$. With these settings, the total linear and angular momenta are initially zero, and the temperature is initially the target temperature. The initial value of the extended variable was set to $\mathrm{v}(0)=0$.

(2) Bulk argon system. The interactions of argon atoms are defined by a pairwise Lennard-Jones type potential with the smooth force-switching scheme of the form,

$$
U(r)=\left\{\begin{array}{ccc}
A r^{-12}-B r^{-6}+a_{0} & \text { for } & 0<r \leq r_{1}, \\
a_{0}+\sum_{k=0}^{4} b_{k} r^{k} & \text { for } & r_{1}<r<r_{\mathrm{c}} \\
0 & \text { for } & r_{\mathrm{c}} \leq r<\infty
\end{array}\right.
$$

where $A=2508000 \AA^{12} \mathrm{kcal} / \mathrm{mol}$ and $B=1545 \AA^{6} \mathrm{kcal} / \mathrm{mol}$. The original force function is defined for $r \leq r_{1}$ and it is smoothly damped zero at the cutoff length $r_{\mathrm{c}}$, where $r_{1}=8 \AA$ and $r_{\mathrm{c}}=10 \AA$ were used. The parameters, $a_{k}$ and $b_{k}$, are determined so as $U$ to be a smooth function, e.g., $a_{0}=-\sum_{k=0}^{4} b_{k} r_{c}^{k}$ (see Ref. 43 for the details). 168 argon atoms were treated within a $20 \times 20 \times 20 \AA^{3}$ cubic box under $3 \mathrm{D}$ periodic boundary 
conditions. The target temperature $T_{0}$ was $87 \mathrm{~K}$, which corresponds to a liquid phase. The initial velocities were set randomly, modified to zero the total linear momentum, and scaled to obtain an initial kinetic energy equal to the target kinetic energy. The initial value of the extended variable was set to $\mathrm{v}(0)=0$.

(3) Bulk ethane system. The intramolecular interaction is the same as in (1), and the intermolecular interactions are defined by a pairwise potential of the same form as (46) with $A=6020089 \AA^{12} \mathrm{kcal} / \mathrm{mol}, B=2165 \AA^{6} \mathrm{kcal} / \mathrm{mol},{ }^{48)} r_{1}=12 \AA$, and $r_{\mathrm{c}}=14 \AA .588$ molecules were treated within a $30 \times 30 \times 30 \AA^{3}$ cubic box under $3 \mathrm{D}$ periodic boundary conditions. $T_{0}$ was $184 \mathrm{~K}$. The initial velocities were set randomly, modified to zero the total linear momentum, and scaled to obtain an initial kinetic energy equal to the target value. The extended variable was set to $\mathrm{v}(0)=0$.

\subsection{Results and discussion}

\subsubsection{Isolated molecule}

To investigate fundamental properties of the integrators, we first apply them to a simple model system, an isolated ethane molecule. We have studied the following four properties: (i) temperature control ability, (ii) accuracy, (iii) robustness, and (iv) time reversibility.

(i) Since this system is small, the temperature fluctuations should be large and the temperature control will not be trivial. To see this, we have varied the value of the temperature-control time constant $\tau$. In general, a small $\tau$ increases the temperature controlling speed but introduces stiffness in the system. In contrast, a large $\tau$ decreases the controlling speed and results in no temperature control in the Newtonian limit $\tau \rightarrow$ $\infty$. Figure 1(a) shows averaged time development of the temperature obtained by the four integration methods using $h=1$ fs and several $\tau$ values. Here, to properly capture the control ability, simple moving average, $T_{\mathrm{MA}}(n) \equiv \frac{1}{N_{\mathrm{MA}}} \sum_{i=1}^{N_{\mathrm{MA}}} T(n-i+1)$, is depicted for $n \geq N_{\mathrm{MA}} \equiv 1000$, instead of the instantaneous temperature at time $t=n h, T(n)$ (see Fig. S1 in Supplementary material ${ }^{40)}$ for the instantaneous temperature). Method 1 was not satisfactory for this system. For smaller $\tau$, the fluctuations are enormously large, and for larger $\tau$ the fluctuations are smaller but the averaged temperature is too high compared with the target temperature, $T_{0}$. Method $1 \bmod$ was better than Method 1 , suggesting that the velocity scaling using the boosted velocity $\tilde{v}=v+h F(x) \mathbf{M}^{-1}$ is better than using the original velocity $v$ [viz., $\lambda_{h}(\tilde{v}) \tilde{v}$ was better than $\lambda_{h}(v) \tilde{v}$ in $\left.(2)\right]$. However, the averaged temperatures of Method $1 \bmod$ are still far from $T_{0}$ for both the 

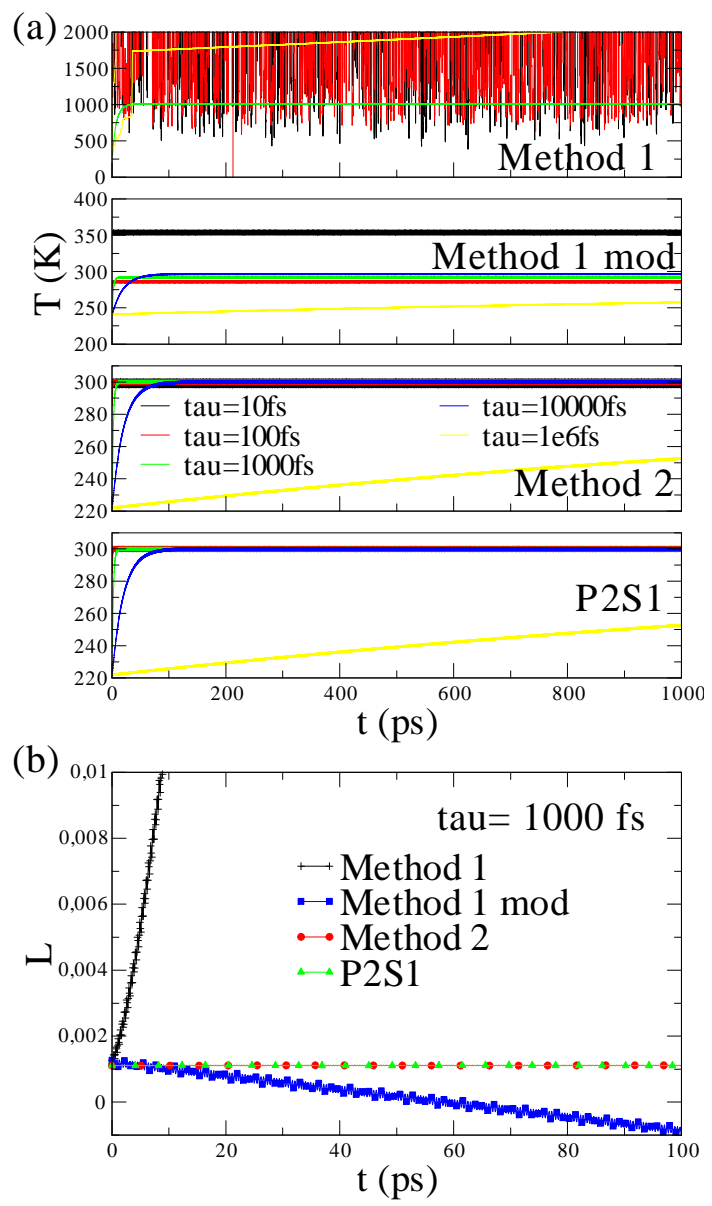

Fig. 1. (a) Trajectories of averaged temperature, $T_{\mathrm{MA}}$, for a single ethane molecule system obtained by four integration methods: Method 1, Method 1 mod, Method 2, and P2S1. Temperature-control time constant $\tau$ is varied, while the unit timestep $h$ is $1 \mathrm{fs}$. (Color figure online) (b) Trajectories of the invariant function $L\left(\AA^{2} \mathrm{~g} / \mathrm{mol} \mathrm{fs}^{-2}\right)$ using the four methods with $h=1$ fs and $\tau=1$ ps.

largest and the smallest $\tau$. Method 2 and P2S1 are comparable, and the temperature control is good except for the largest $\tau$. The smaller the $\tau$ the faster the control becomes, as theoretically expected, and it does work even for the smallest $\tau$.

(ii) We have checked the numerical error by the conservation of the invariant function defined by (16) applying (14). Figure 1(b) shows the trajectory of the invariant $\left(\AA^{2} \mathrm{~g} / \mathrm{mol} \mathrm{fs}^{-2}\right)$ obtained by each method with $h=1$ fs and $\tau=1 \mathrm{ps}$. The drift in Method 1 is significant, which may be expected from the temperature uncontrollability as discussed above. Method 1 mod also exhibits unignorable drift, although the temperature control is relatively good for these $h$ and $\tau$ values as shown in Fig. 1(a). This indicates that the judgement of the simulation validity only by the temperature controllability is in fact insufficient. Method 2 and P2S1 are comparable and show good 

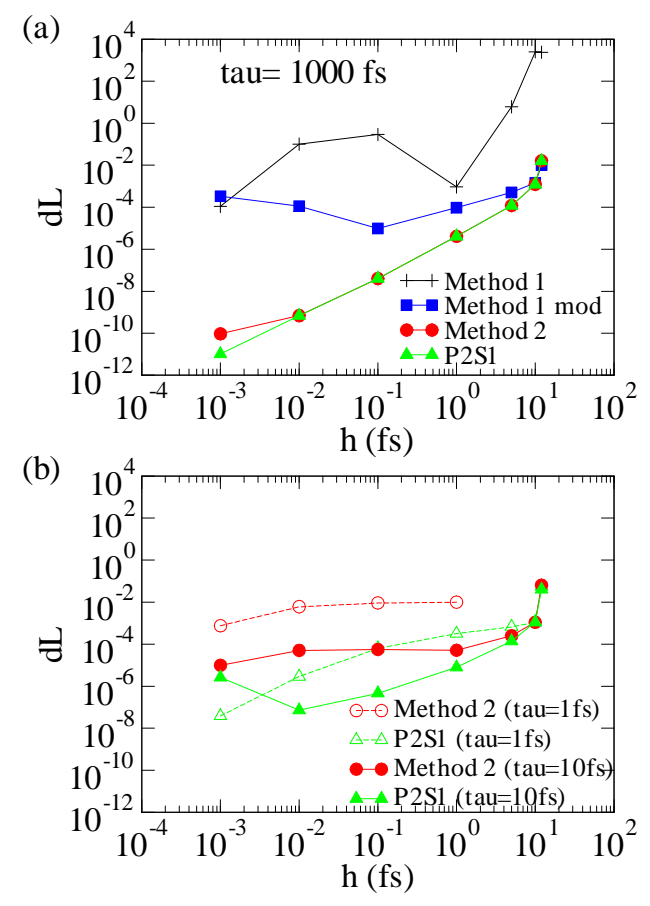

Fig. 2. The errors of the invariant function obtained for the single ethane molecule system. Unit timestep $h$ is varied. (a) Four integration methods using $\tau=1000$ fs. (b) Method 2 and P2S1 using $\tau=1$ or 10 fs.

conservations of the invariant.

To investigate the accuracy in detail, the behavior of the trajectory of $L$ needs to be captured properly. The error of the invariant $d L$ is obtained by the following formula: 43 )

$$
d L=\left.\left\langle\left.\left\langle\left|L(t)-L\left(t_{0}\right)\right|\right\rangle\right|_{t=t_{0}} ^{t_{0}+\mathrm{sd}}\right\rangle\right|_{t_{0}}
$$

where one thousand different time origins, $t_{0}$, have been chosen randomly, and the sampling duration, sd, was 10 ps. Figure 2(a) shows the error $d L$ computed for various values of timestep $h$. Method 1 generates large errors especially for $h>1$ fs. Method 2 and P2S1 are comparable (when a relatively large $\tau$ is used) and imply good second-order integrator behavior. ${ }^{43)}$ Their difference appears for smaller $h$ values (and for smaller $\tau$ values as detailed below).

Although Method 2 is not considered to be an exact second-order map, it includes the $\Phi_{h / 2}^{[2]} \circ \Phi_{h}^{[1]} \circ \Phi_{h / 2}^{[2]}$ sequence, which corresponds to the second order map for the Newtonian part of the EOM. This is not the case for Method 1 and Method 1mod. Under "mild" conditions, i.e. when $K(v) \sim K_{0}$ (near equilibrium) and/or when $s \equiv h / \tau$ is sufficiently small (non "stiff"), it may hold that $\Phi_{\mathrm{M} 2, h} \sim \Psi_{h}^{\mathrm{P} 2 \mathrm{~S} 1}+\mathcal{O}\left(s^{3}\right)$ or a weaker relationship such as $\Phi_{\mathrm{M} 2, h} \sim \varphi_{h} \circ \Psi_{h}^{\mathrm{P} 2 \mathrm{~S} 1} \circ \varphi_{h}^{-1}+\mathcal{O}\left(s^{3}\right)$ for a certain invertible map $\varphi_{h}$ 
(viz., $\varphi_{h}$ becomes a postprocessor ${ }^{49)}$ between $\Psi_{h}^{\mathrm{P} 2 \mathrm{~S} 1}$ and $\Phi_{\mathrm{M} 2, h}$ in an approximate sense). These relationships suggest a second-order like property for $\Phi_{\mathrm{M} 2, h}$.

(iii) The robustness (stated in (iii-1) of Sect. 4.3 ) of the proposed method, compared with Method 1 and Method 1mod, is well illustrated in Figs. 1 and 2. The indication on issue (iii-1) really makes sense, because the dynamics generated by P2S1 using $\Lambda_{h}(v)$ encounter similar or smaller fluctuations of $K(v)$ than the dynamics generated by Method 1, Method 1 mod, and Method 2, all of which use $\lambda_{h}(v)$.

The robustness of P2S1 in the sense of (iii-2) in Sect. 4.3, due to the difference between $\lambda_{h}(v)$ and $\Lambda_{h}(v)$ [viz., the difference between $\tilde{\Phi}_{h}^{[3]}$ and $\Phi_{h}^{[3]}$, is demonstrated by increasing $s \equiv h / \tau$. This also reveals the difference between Method 2 and P2S1, as depicted in Fig. 2(b), which shows $d L$ computed with smaller values of $\tau$ than in Fig. 2(a). For $\tau=10$ fs, P2S1 is superior in accuracy to Method 2, although the difference vanishes for larger $h$ because the principal error may come from the common maps $\Phi_{h}^{[2]}$ and $\Phi_{h}^{[1]}$. For $\tau=1$ fs, the difference between Method 2 and P2S1 is even larger, and Method 2 broke for $h>1$ fs, due to the domain exception problem (see Appendix for details).

(iv) Figure 3 shows the results of a time reversing test. After a "forward" simulation for $M=100$ time steps with unit timestep $h=1 \mathrm{fs}$, it was changed into the negative value, viz., $h=-1$ fs, and a "backward" simulation was conducted for $M$ time steps. If the trajectory is exact, then we will have the same $(x, v)$ value at $M-m$ time step and at $M+m$ time step for every $m=1, . ., M$. They were deviated much for Method 1 , as indicated in Fig. 3(a). Furthermore, it broke at $M+21$ time step due to the use of negative timestep (see Appendix). For Method 2 [Fig. 3(b)], the correspondence between $M-m$ and $M+m$ is better, but the deviations were gradually increased and also resulted in a break at $M+67$ steps. In contrast, the correspondence is almost perfect for P2S1 and resulted in the same $(x, v)$ value at the final $2 M$ time steps as the initial value [Fig. 3(c)]. These results clearly indicate that the current P2S1 method is really time reversible, which is a fundamental property of the original ODE and should be possessed by accurate numerical integrators. Note that "backward" simulation is not done in ordinary simulations, but it is preferable to have many measures, including the "backward" simulation analysis, to detect numerical errors, where the loss of the time reversibility indicates potential errors. 

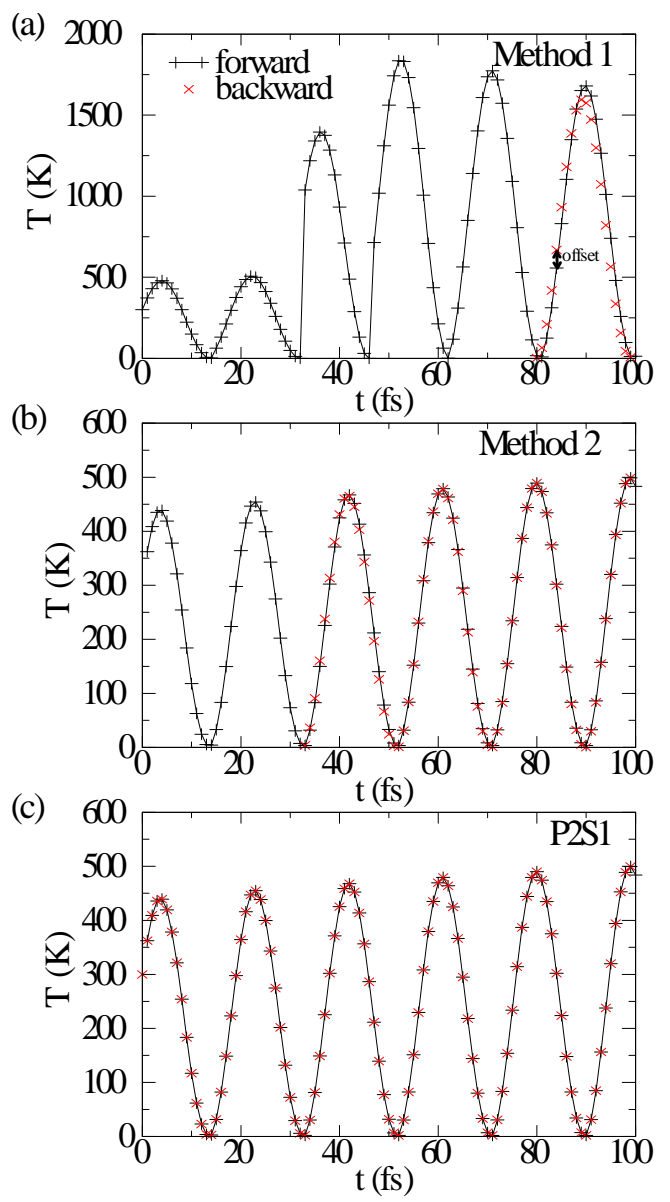

Fig. 3. The reversibility for (a) Method 1, (b) Method 2, and (c) P2S1, tested on the single ethane molecule system. Black "+" designates the temperature of each timestep obtained in the forward simulation, while red " $\times$ " the backward simulation. "Offset" shows the deviation between the forward and backward values.

\subsubsection{Bulk argon}

Figure 4(a) shows the trajectories of the averaged temperature $T_{\mathrm{MA}}$ in the bulk argon system using $h=1$ fs. Temperature control ability is similar for all the methods. In each case, a smaller $\tau$ induces a fast control, while a larger $\tau$ leads to a slow control, as expected. The long-time averages of the temperatures are also similar for all the four methods and are near to the target temperature, except for a very large $\tau$, as shown in Fig. S2. These resemblances are in contrast to the smaller system, the isolated molecule, discussed above. However, the accuracy measured by the invariant deviation $d L$ clarified the difference between the methods. Figure 4(b) shows $d L$, which was estimated in the same manner as the isolated molecule system, by using several $h$ values and a fixed $\tau$ value at 1 ps. The accuracies of P2S1 and Method 2 are at least one order of magnitude 

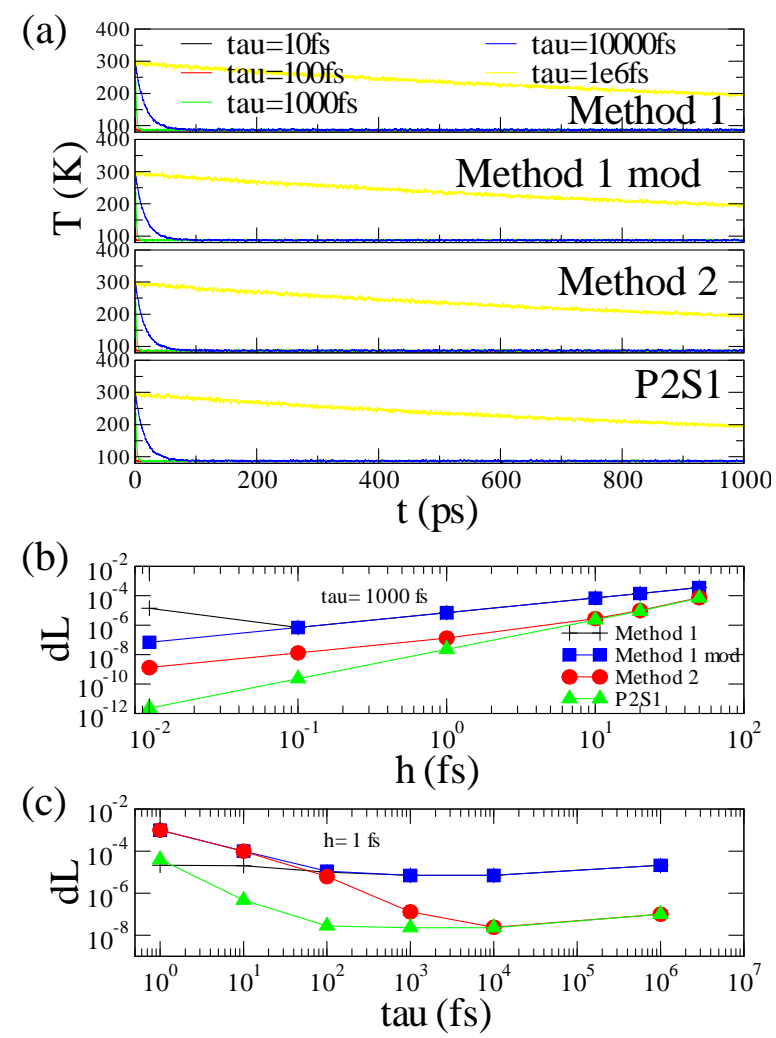

Fig. 4. (a) Trajectories of the averaged temperature for a bulk argon system obtained by Method 1, Method 1 mod, Method 2, and P2S1. Temperature-control time constant $\tau$ is varied. (Color figure online) The errors of the invariant function are shown with (b) unit timestep $h$ being varied (abscissa) with $\tau$ fixed to be $1 \mathrm{ps}$, and (c) $\tau$ being varied (abscissa) with $h$ fixed to be 1 fs.

better than that of Method 1 and Method 1 mod. P2S1 is always the most accurate method and the difference between P2S1 and other methods increases with decreasing $h$ (Fig. 4(b)) and decreasing $\tau$ (Fig. 4(c)). That is to say, P2S1 is much more accurate than the other methods when one seeks for a fast temperature control. As shown in Fig. 4(c), P2S1 is more accurate, by one or two orders of magnitude, than Method 2 for $\tau \lesssim 1000$ fs with a fixed $h$ value at 1 fs. These results are similar when larger $h$ are used, as shown in Fig. S3 in Supplementary material. ${ }^{40)}$

\subsubsection{Bulk ethane}

The robustness of the proposed method was also observed in the bulk ethane system, as shown in Fig. 5. For $\tau=1$ ps, the accuracies of the two methods, P2S1 and Method 2, are one or more orders of magnitude better than that of the remaining two methods, Method 1 and Method 1 mod. However, for $\tau=10$ fs, the situation clearly changed, and only P2S1 is accurate, with two or more orders of magnitude, compared with Method 1, 

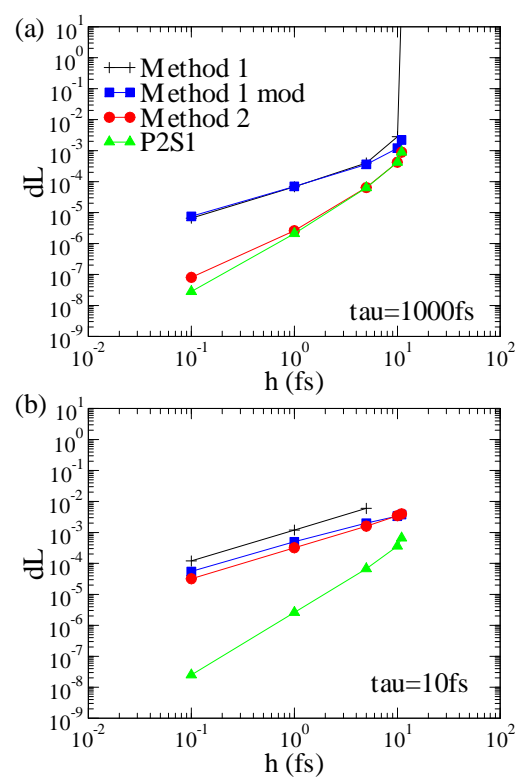

Fig. 5. The errors of the invariant function obtained for a bulk ethane system using the four integration methods. Unit time step $h$ is varied with $\tau$ fixed to be (a) 1 ps and (b) 10 fs.

Method 1 mod, and Method 2 for a wide range of $h$. The maximum $h$ value for P2S1 with the $\tau=10$ fs case was 11 fs.

Remarks regarding the $d L$ vs. $h$ curves are made. These curves are very smoother than those obtained in the single molecule system indicated in Fig. 2(a). This should be related to the fact that the temperature deviation of the small system is large (see Fig. S1 of Supplementary material ${ }^{40)}$ ) so that the temperature control is difficult and the system becomes stiff. On the other hand, the gradient of the curve is considered to correspond the local order of the accuracy. ${ }^{43)}$ In this viewpoint, Method 2 seems to be a second-order integrator if $\tau=1000 \mathrm{fs}$ [Fig. 5(a)] but it seems to be a first-order integrator if $\tau=10$ fs [Fig. 5(b)]. Thus, Method 2 is implied to have a intermediate property between these two accuracies.

\section{Conclusion}

The proposed scheme for numerically integrating the Berendsen temperature-control EOM works well with respect to the temperature-control ability, accuracy, robustness, and time reversibility. It was analyzed theoretically and examined numerically by simulating an isolated ethane molecule, a bulk argon system, and a bulk ethane system.

The superiority of the proposed integrator in the temperature-control ability is particularly clear in a stiff system, which is here an isolated molecule system having large 
fluctuations. The accuracy and robustness have also been demonstrated with the two bulk systems. The proposed P2S1 integrator and the conventional integrators are equivalent in the computational cost and the first-order local accuracy. But P2S1 is second order, and also the proposed scheme enables us to attain higher accuracies. The accuracy measured by the invariant function for P2S1 was one or two orders of magnitude better than that of the conventional integrators, for wide range of timestep values $h$ and in particular for small values of the temperature-control time parameter, $\tau$.

Even though the statistical ensemble for the Berendsen EOM is not properly defined, the Berendsen method has been widely used for molecular simulations because of its robustness. The interesting point of the proposed scheme is that it can make the method even more robust. This scheme is particularly superior when one seeks a fast temperature control, by using a large $h$ or a small value of $\tau$. The robustness of the proposed method originates from the velocity scale factor $\Lambda_{h}(v)$, which is a counterpart of $\lambda_{h}(v)$ used in conventional methods. Although a suitable $\tau$ value may depend on physical consideration or simulation purpose, the results provided by the proposed method were similar or superior than those of the conventional methods for all $\tau$ values investigated.

The proposed operator-map scheme is successful to capture the property of the original ODE, the time reversibility. The time reversibility of the proposed method comes from a suitable decomposition of the Berendsen vector field and the symmetric composition technique of the resultant exact maps. The time reversibility, or symmetric property, in an integrator map is explicitly useful for a vector field having the $\rho$-reversibility, because it conserves the $\rho$-reversibility for the exact flow in many cases. Since the Berendsen vector field does not seem to admit a $\rho$-reversibility in general, the current positive results on the P2S1 integrator suggests that the time reversibility is also useful for a vector field that has no $\rho$-reversibility.

In this study, we restrict our attention on the most fundamental scheme, P2S1, among the proposed methods. This is because it is simply implemented while highly effective. Higher-order integration schemes, such as fourth-order integrators P4S5 and P4S6, can be used according to the proposed method, in order to attain higher accuracy for a small $h$. For a larger $h$, an alternative second-order integrator, P2S2, is useful and will show a comparable or better performance relative to P2S1.

We also showed that a good performance of the numerical integrator was not measured only by the temperature-control ability. The currently proposed invariant function 
defined on the extended phase space is useful for any integrator to detect the numerical error and to prevent unphysical results.

\section{Acknowledgements}

This work was supported by a Grant-in-Aid for Scientific Research (C) (25390156 and 17K05143) from JSPS and by Japan Agency for Medical Research and development, AMED. The part of the work was performed during a stay of Q.S. in Osaka University by the support of the International Joint Research Promotion Program.

\section{Appendix}

Here we analyze and compare the two velocity scale factors:conventional one, $\lambda_{h}(v)$, and the currently introduced one, $\Lambda_{h}(v)$.

\subsection{Basics}

To simplify the discussion, we re-parametrize these factors, using $s \equiv h / \tau \in \mathbb{R}$ (the ratio of the timestep to the time constant) and $r \equiv K_{0} / K(v) \in \mathbb{R}_{+}$(the ratio of the target value to the instantaneous value, with respect to the kinetic energy; $\mathbb{R}_{+}$denotes strictly positive numbers), such that

$$
\begin{aligned}
& \lambda(r, s)=[1+s(r-1)]^{\frac{1}{2}}, \\
& \Lambda(r, s)=[(1-r) \exp (-s)+r]^{\frac{1}{2}},
\end{aligned}
$$

reducing $\lambda\left(K_{0} / K(v), h / \tau\right)=\lambda_{h}(v)$ and $\Lambda\left(K_{0} / K(v), h / \tau\right)=\Lambda_{h}(v)$. Since we need realvalued factors, first we should clarify the domain of definition of $\lambda$ and that of $\Lambda$. They are, respectively, given by

$$
\begin{aligned}
& \Gamma_{\lambda} \equiv\left\{(r, s) \in \mathbb{R}_{+} \times \mathbb{R} \mid s \leq s_{\lambda}(r) \text { if } r<1,\right. \\
& \text { and } \left.s \geq s_{\lambda}(r) \text { if } r>1\right\}, \\
& \Gamma_{\Lambda} \equiv\left\{(r, s) \in \mathbb{R}_{+} \times \mathbb{R} \mid s \geq s_{\Lambda}(r) \text { if } r>1\right\}
\end{aligned}
$$

[see Figs. 6 (a) and (b)], where

$$
\begin{aligned}
& s_{\lambda}(r) \equiv(1-r)^{-1} \\
& s_{\Lambda}(r) \equiv \ln \left(1-r^{-1}\right) .
\end{aligned}
$$

\subsubsection{Domain exception problem}

The integrator breaks down if $\lambda$ or $\Lambda$ takes a value out of $\Gamma_{\lambda}$ or $\Gamma_{\Lambda}$. 


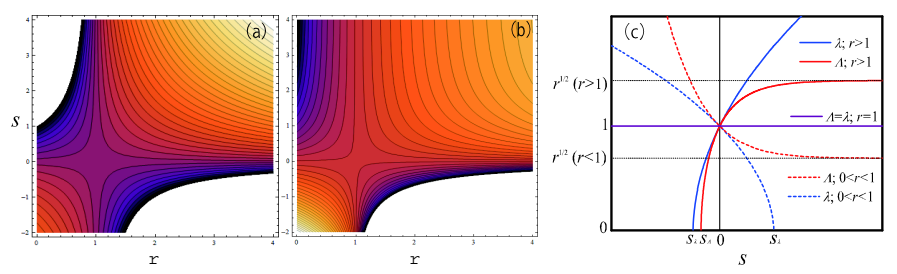

Fig. 6. Velocity scale factors $\lambda(r, s)=[1+s(r-1)]^{\frac{1}{2}}$ and $\Lambda(r, s)=[(1-r) \exp (-s)+r]^{\frac{1}{2}}$, where $r \equiv K_{0} / K(v)$ and $s \equiv h / \tau$. Domains of definition of (a) $\lambda$ and (b) $\Lambda$ are shown, as well as contours (large as light color). (c) Values of the factors with respect to $s$, where $r \equiv 2>1, r \equiv 1$, and $r \equiv 0.5<1$ cases are indicated (Color figure online).

$(r=1$ case $)$ Since

$$
\lambda(1, s)=\Lambda(1, s)=1
$$

holds for all $s \in \mathbb{R}$, there is no problem in this case, as expected from the fact that $r=1$ means the "equilibrium" $K_{0}=K(v)$.

$(r<1$ case) If $r<1$, then admissible $s$ for $\lambda(r, s)$ is restricted such that $s \leq$ $s_{\lambda}(r)$, but this condition is met as long as $s \leq 1$, namely, $h \leq \tau$. Thus, the domain exception problem may not be severe in many practical simulations (nevertheless, we have confirmed this problem for Method 2 in Fig. 2(b)). In contrast, since all $s \in \mathbb{R}$ is admissible for $\Lambda(r, s)$ when $r<1$, no problem arrises in the proposed method.

$(r>1$ case) If $r>1$, then every $s \geq 0$ is admissible for both $\lambda$ and $\Lambda$ [since $s_{\lambda}(r), s_{\Lambda}(r)<0$ if $r>1$ ], while the use of negative $s$ is restricted. We have encountered this type of domain exception in the backward simulation for the conventional methods as seen in Figs. 3(a) and 3(b). In contrast, for the current method using $\Lambda$ in $\Phi_{h}^{[3]}$, the usual group property, $\left(\Phi_{-h}^{[3]} \circ \Phi_{h}^{[3]}\right)\left(\omega^{\prime}\right)=\omega^{\prime}$, ensures no exception in the backward simulation. However, this type of the domain exception concerns higher-order integrators (see Sect. 7.3).

\subsubsection{Graph}

Both $\lambda$ and $\Lambda$ are monotonic functions with respect to both $r$ and $s$ (strictly monotonic unless $r=1$ or $s=0$ ). Typical graphs are depicted in Fig. 6(c). Equation (34) can be obtained (see also Remark 9 of Supplementary material ${ }^{40)}$ ) from the Maclaurin's expansions of $\lambda$ and $\Lambda$ with respect to $s$ such that

$$
\Lambda(r, s)=1+\frac{1}{2}(r-1) s-\frac{1}{8}\left(r^{2}-1\right) s^{2}+\mathcal{O}\left(s^{3}\right),
$$




$$
\lambda(r, s)=1+\frac{1}{2}(r-1) s-\frac{1}{8}(r-1)^{2} s^{2}+\mathcal{O}\left(s^{3}\right) .
$$

Note that $\Lambda(r, s) \neq \lambda(r, s)+\mathcal{O}\left(s^{3}\right)$ in general (the equality holds only in the "equilibrium" case $r=1$ ).

\subsection{The statements on the robustness}

We mathematically formulate the statements on issues (iii-1) and (iii-2) in Sect. 4.3 as Propositions 2 and 3, respectively. Proofs of propositions are given in Sect. III of Supplementary material. ${ }^{40)}$

Issue (iii-1)

When $r=1$, viz., kinetic energy $K(v)$ takes the target value $K_{0}$, then the factors do nothing, that is, (53) holds. In simulations, $r$ varies so that $\lambda$ and $\Lambda$ fluctuate around the unity. As a fundamental property, we observe

Proposition 1 (a) If $0<r<1$ then $\lambda(r, s)=\Lambda(r, s)=1$ for $s=0$ and $\lambda(r, s)<$ $\Lambda(r, s)$ for all admissible $s \neq 0$, (b) if $r=1$ then $\lambda(r, s)=\Lambda(r, s)=1$ for all $s \in \mathbb{R}$, and (c) if $r>1$ then $\lambda(r, s)=\Lambda(r, s)=1$ for $s=0$ and $\lambda(r, s)>\Lambda(r, s)$ for all admissible $s \neq 0$.

Now, we see that the amplitude of $\lambda(r, s)$ from its "equilibrium" value 1 is larger than that of $\Lambda(r, s)$ :

Proposition $2|\lambda(r, s)-1| \geqq|\Lambda(r, s)-1|$ holds for any $(r, s) \in \Gamma_{\lambda} \cap \Gamma_{\Lambda}$ provided that $s>0$, where the equality holds only if $r=1$.

\section{Issue (iii-2)}

Consider the behavior of $\lambda(r, s)$ and $\Lambda(r, s)$ as increasing $s=h / \tau$, viz., increasing the unit timestep $h$ or decreasing the temperature-control time constant $\tau$. As for a global behavior, $\Lambda(r, s)$ is bounded for $s \geq 0$ and $\lim _{s \rightarrow \infty} \Lambda(r, s)=r^{\frac{1}{2}}$ for any $r>0$, but $\lambda(r, s)$ is not. In fact, $\lambda(r, s)$ is unbounded and $\lim _{s \rightarrow \infty} \lambda(r, s)=\infty$ if $r>1$, and $\lambda(r, s)$ cannot be defined anymore for $s>s_{\lambda}(r)$ if $r<1$. This implies that $\lambda(r, s)$ is not tractable for increasing $s$. These differences of the global behavior between $\lambda(r, s)$ and $\Lambda(r, s)$ are in contrast to the similarities of the local behavior between them, as seen in (54). The importance in practice may be in the middle range of $s$. As expected from these facts, the behavior of the difference between $\lambda(r, s)$ and $\Lambda(r, s)$ is described as follows, which now expresses the statement in (iii-2):

Proposition $3|\lambda(r, s)-\Lambda(r, s)|$ is strictly monotone increasing with respect to $s$ for 
any admissible $s>0$ and for an arbitrarily fixed $r \in \mathbb{R}_{+} \backslash\{1\}$.

\subsection{Robust higher-order method}

For constructing a robust higher-order integration method, we should overcome the problem originated from the fact that $\Lambda_{h}(v)$ does not permit an arbitrary $h \in \mathbb{R}$. Namely, the domain of the definition of $\Lambda(r, s)$ for a fixed $r$ is $\left[s_{\Lambda}(r), \infty\right) \varsubsetneqq \mathbb{R}$ if $r>1$, while it is whole $\mathbb{R}$ if $0<r \leq 1$. From this fact, one cannot use arbitrary negative coefficients $\left\{\alpha_{i}, \beta_{i}\right\}$ in (32), since $\alpha_{i} h$ and $\beta_{i} h$ play a role of an intermediate unit timestep and require the evaluation of $\Lambda_{\alpha_{i} h}(v)$ and $\Lambda_{\beta_{i} h}(v)$. One way to solve this problem is to use a higher-order integrator whose coefficients are all positive. ${ }^{50-52)}$ The other way is to use, instead of $\Lambda(r, s)$, its suitable approximation, $\hat{\Lambda}(r, s)$, which is defined for all $h \in \mathbb{R}$ to enable us to use any negative coefficients. The map $\hat{\Phi}_{h}^{[3]}$, which uses $\hat{\Lambda}$ instead of $\Lambda$, should also be 1-1 for all $h$, in order to construct its adjoint map. 


\section{References}

1) W. G. Hoover, Computational Statistical Mechanics (Elsevier, Amsterdam, 1991).

2) R. Skeel, SIAM J. Sci. Comput. 31, 1363 (2009).

3) M. Allen and D. Tildesley, Computer Simulation of Liquids (Oxford, New York, 2002).

4) T. Schlick, Molecular Modeling and Simulation: an Interdisciplinary Guide (Springer Science \& Business Media, New York, 2010), Vol. 21.

5) S. Nosé, Progress of Theoretical Physics Supplement 103, 1 (1991).

6) P. H. Hüenberger, in Advanced Computer Simulation: Approaches for Soft Matter Sciences I., edited by C. Holm, and K. Kremer (Springer, Berlin, 2005), p. 105.

7) O. G. Jepps and L. Rondoni, J. Phys. A: Math. Theor. 43, 133001 (2010).

8) S. Nosé, J. Chem. Phys. 81, 511 (1984).

9) W. Hoover, Phys. Rev. A 31, 1695 (1985).

10) S. Pieprzyk, D. M. Heyes, S. Maćkowiak, and A. C. Brańka, Phys. Rev. E 91, 03331 (2015).

11) I. Fukuda and K. Moritsugu, Phys. Rev. E 93, 033306 (2016).

12) B. Leimkuhler and X. Shang, SIAM J. Sci. Comput. 38, A712 (2016).

13) I. Fukuda, Phys. Lett. A 380, 2465 (2016).

14) W. G. Hoover, J. C. Sprott, and P. K. Patra, Phys. Lett. A 379, 2935 (2015).

15) W. G. Hoover, Molecular Dynamics (Springer-Verlag, Berlin, 1986).

16) W. G. Hoover, A. J. C. Ladd, and B. Moran, Phys. Rev. Lett. 48, 1818 (1982).

17) D. J. Evans, J. Chem. Phys. 78, 3297 (1983).

18) D. J. Evans, W. G. Hoover, B. H. Failor, B. Moran, and A. J. C. Ladd, Phys. Rev. A 28, 1016 (1983).

19) D. J. Evans and G. P. Morriss, Phys. Lett. A 98, 433 (1983).

20) H. J. C. Berendsen, J. P. M. Postma, W. F. van Gunsteren, A. DiNola, and J. R. Haak, J. Chem. Phys. 81, 3684 (1984).

21) H. J. C. Berendsen, D. van der Spoel, and R. van Drunen, Comput. Phys. Commun. 91, 43 (1995).

22) H. Eslami, F. Mojahedi, and J. Moghadasi, J. Chem. Phys. 133, 084105 (2010). 
23) S. A. Moga, N. Goga, and A. Hadar, Materiale Plastice 50, 196 (2013).

24) T. Morishita, J. Chem. Phys. 113, 2976 (2000).

25) G. Bussi, D. Donadio, and M. Parrinello, J. Chem. Phys. 126, 014101 (2007).

26) J. E. Basconi and M. R. Shirts, J. Chem. Theory Comput. 9, 2887 (2013).

27) D. Okunbor and R. D. Skeel, Mathematics of Computation 59, 439 (1992).

28) J.-M. Sanz-Serna and M.-P. Calvo, Numerical Hamiltonian problems, volume 7 of Applied Mathematics and Mathematical Computation (Chapman \& Hall, London, 1994).

29) R. D. Ruth, IEEE Trans. Nucl. Sci 30, 2669 (1983).

30) H. Yoshida, Phys. Lett. A 150, 262 (1990).

31) R. I. McLachlan and P. Atela, Nonlinearity 5, 541 (1992).

32) J. M. Sanz-Serna, Acta Numerica 1, 243 (1992).

33) R. Skeel, G. Zhang, and T. Schlick, SIAM J. Sci. Comput. 18, 203 (1997).

34) M. Tuckerman, B. J. Berne, and G. J. Martyna, J. Chem. Phys. 97, 1990 (1992).

35) A. Dullweber, B. Leimkuhler, and R. McLachlan, J. Chem. Phys. 107, 5840 (1997).

36) I. P. Omelyan, I. M. Mryglod, and R. Folk, Phys. Rev. Lett. 86, 898 (2001).

37) I. Fukuda and H. Nakamura, Phys. Rev. E 73, 026703 (2006).

38) R. I. McLachlan and G. Quispel, Six lectures on the geometric integration of ODEs (School of Mathematical and Statistical Sciences, La Trobe University, 1998).

39) R. I. McLachlan and G. R. W. Quispel, Acta Numerica 11, 341 (2002).

40) (Supplemental material) Details of the algorithm and results, as well as mathematical proofs for the propositions and the accuracies, are provided online.

41) V. L. Golo and K. V. Shaitan, Biophysics 47, 567 (2002).

42) M. Khalili, A. Liwo, A. Jagielska, and H. A. Scheraga, J. Phys. Chem. B 109, 13798 (2005).

43) S. Queyroy, H. Nakamura, and I. Fukuda, J. Comput. Chem. 30, 1799 (2009).

44) E. Hairer, C. Lubich, and G. Wanner, Geometric numerical integration: structurepreserving algorithms for ordinary differential equations (Springer-Verlag, Berlin, 2002).

45) R. I. McLachlan, SIAM J. Sci. Comput. 16, 151 (1995). 
46) S. Blanes and P. C. Moan, J. Comput. Applied Math. 142, 313 (2002).

47) S. C. Harvey, R. K. Z. Tan, and T. E. Cheatham, J. Comput. Chem. 19, 726 (1998).

48) M. G. Martin and J. I. Siepmann, J. Phys. Chem. B 102, 2569 (1998).

49) S. Blanes, F. Casas, and A. Murua, SIAM Journal on Numerical Analysis 42, 531 (2004).

50) M. Suzuki, Phys. Lett. A 201, 425 (1995).

51) S. A. Chin, Phys. Lett. A 226, 344 (1997).

52) J. Auer, E. Krotscheck, and S. A. Chin, J. Chem. Phys. 115, 6841 (2001). 